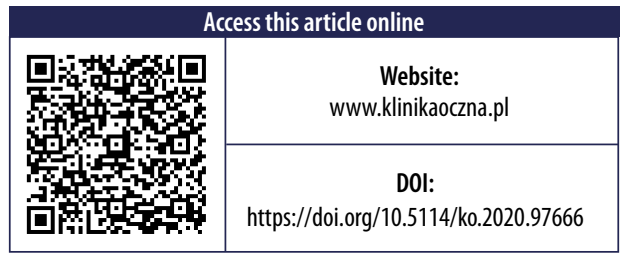

\title{
Inter-eye asymmetry in manifest refraction, keratometry and pachymetry in eyes with keratoconus
}

\author{
Magdalena Maleszka-Kurpiel ${ }^{1,2}$, Andrzej Michalski ${ }^{3}$, Marta Robak', Wojciech Warchoł² \\ 'Optegra Eye Health Care Clinic, Poznan, Poland \\ 2Department of Optometry, Chair of Ophthalmology and Optometry, Poznan University of Medical Sciences, Poznan, Poland \\ ${ }^{3}$ Department of Ophthalmology, Chair of Ophthalmology and Optometry, Poznan University of Medical Sciences, Poznan, Poland
}

\begin{abstract}
Aim of the study: To evaluate selected visual system parameters in keratoconus patients and establish criteria which will enable better screening for keratoconus.

Material and methods: 146 eyes of 73 patients diagnosed with keratoconus were included in the study. Each patient underwent optometric and ophthalmological examination with corneal tomography.

Results: We found a statistically significant inter-eye difference between the better and worse eye in median values of uncorrected (0.24 vs. $0.62 \operatorname{LogMAR}$ ) and best corrected (0.03 vs. 0.24 LogMAR) distance visual acuity. Our study also showed statistically
\end{abstract}

significant differences in median values of keratometry between the better and worse eye (K1 43.1 vs. $45.4 \mathrm{D}, \mathrm{K} 245.2$ vs. $49.0 \mathrm{D}$ ), mean values of thinnest central cornea (TCC) ( $488 \mathrm{vs.} 458 \mu \mathrm{m})$ and median grade 1.5 and 2.5 for the better and worse eye, respectively. The most prevalent refractive error was compound myopic astigmatism, followed by hyperopic compound or mixed astigmatism. Conclusions: Keratoconus should be suspected in patients with inter-eye differences in manifest refraction, especially when astigmatism exists. Any inter-eye asymmetry in keratometry or pachymetry values should be an indication for full keratoconus screening. KEY WORDS: keratoconus, inter-eye asymmetry, pachymetry, keratometry, manifest refraction.

\section{INTRODUCTION}

Keratoconus $(\mathrm{KC})$ is a progressive corneal, bilateral ectasia characterized by thinning and weakening of the cornea that results in corneal steepening, protrusion, irregular astigmatism, and gradual impairment of vision [1].

Worldwide, KC occurs in approximately 1 in 2000 individuals, as reported by Rabinowitz at the end of the $20^{\text {th }}$ century [1]. However, reported epidemiological data differ between geographical zones and other factors such as age or gender.

Ethnicity has been reported to play a role in keratoconus. Asians have 4.4 times higher risk for developing keratoconus than Caucasians, and Indians have steeper corneas than Chinese patients with keratoconus [2,3]. Recently reported $\mathrm{KC}$ prevalence in the pediatric population in Saudi Arabia is higher than in previous reports: 4790/100,000 (4.79\%) [4].

Keratoconus affects both genders, and data about gender predilection are not consistent. Li et al. found no difference between genders, whereas Wagner et al. found $\mathrm{KC}$ more frequently in males $[5,6]$.

Keratoconus is a multifactorial disease caused by genetic and environmental factors. Genetics of $\mathrm{KC}$ are still under study, but multiple genes have been identified as potential disease risk factors [7-9]. There were studies and anecdotal reports published supporting the idea that in development of $\mathrm{KC}$ mechanical factors such as eye rubbing are involved [10, 11]. Contact lenses, especially rigid gas permeable (RGP), are also considered as a risk factor by causing microtraumas and increased dryness which provokes eye rubbing [12, 13].

Keratoconus has been classified as a noninflammatory disease; however, recent studies found evidence of inflammatory markers, and cytokines including interleukins (IL-1, IL-6, IL-8) and tumor necrosis factor $\alpha$ (TNF- $\alpha$ ) in the tears of patients with keratoconus [14-16].

Coexistence of $\mathrm{KC}$ with systemic conditions is widely discussed. Atopy is found in 53\% of patients with KC [17]. Kaya et al. stated that $\mathrm{KC}$ in patients with atopy differs significantly from $\mathrm{KC}$ in patients without atopy and could be described as a separate clinical condition [18]. Allergy and atopy are reported as dominant risk factors for the habit of eye rubbing [19].

Refractive status of patients with keratoconus is well described. The most common refractive error in $\mathrm{KC}$ is compound myopic astigmatism, with the incidence rate in 
different studies reported from $69.7 \%$ to $85.7 \%$. Types of astigmatism (with-the-rule, against-the-rule, oblique) are different depending on stages of KC [20,21]. Frequency of high astigmatism (>2 D) is significantly higher in population with $\mathrm{KC}$ and subclinical $\mathrm{KC}(14.1 \%)$ than in the normal population (6.86\%) [22-24].

Early diagnosis of keratoconus is still a challenge; the most important aspect is to think about this condition as soon as possible.

Keratoconus can be clinically diagnosed on slit-lamp findings, e.g. corneal thinning, Vogt's striae, Fleischer ring, Munson sight, corneal scarring. However, these changes are seen in severe stages of the disease [1].

The gold standard in $\mathrm{KC}$ diagnosis and monitoring its progression is corneal tomography. But to diagnose the earliest stages of keratoconus also epithelium thickness mapping should be used $[25,26]$.

The management of $\mathrm{KC}$ is mainly consisted of visual acuity improvement using glasses and contact lenses, especially rigid gaspermeable, intracorneal ring segment implantation for moderate stages and keratoplasty (lamellar or penetrating) for advanced ones [27].

A well-described strategy for slowing the progression of keratoconus is to perform corneal collagen cross-linking (CXL). Wollensak reported in 2006 the results of the first clinical study on CXL with riboflavin and UVA as a procedure for the treatment of progressive keratoconus in adults [28]. CXL has been proven to be successful at stiffening and thus arresting and in many cases even regressing the progression [28-30].

The Global Consensus on Keratoconus and Ectatic Diseases, involving opinions from $45 \mathrm{KC}$ clinical experts from around the world, agreed that CXL can be beneficial upon diagnosis in young patients with keratoconus [31].

The keratoconus patient requires a multi-professional approach in which at different stages (clinical suspicion, diagnosis, management and follow-up) optometrists, contact lens practitioners and ophthalmologists are involved.

\section{AIM OF THE STUDY}

The aim of our study was to evaluate and perform a statistical analysis of the selected visual system parameters in keratoconus patients.

\section{MATERIAL AND METHODS}

All study participants were recruited and examined in the Optegra Eye Health Care Clinic in Poznań, Poland. Accurate anamnesis was performed with special attention given to coexisting atopic diseases. Each patient underwent optometric examination including uncorrected distance visual acuity (UCVA), best corrected distance visual acuity (BCVA) (visual acuity was assessed with a Snellen chart and then converted to LogMAR visual acuity). We used manifest refraction for further analysis, and not objective refraction (cycloplegic autorefractometry). Then ophthalmological examination was performed, which included anterior and posterior segment evaluation ( $1 \%$ tropicamide (WZF, Polfa S.A.) was used for pupil dilatation), and intraocular pressure measurement. Imaging examination included corneal tomography WaveLight Oculyzer II (Alcon, Texas, US), and the following parameters were used for further analysis - keratometry: K1 (flat), K2 (steep), grade, thinnest central cornea (TCC). Grade is an automatic classification of keratoconus stage based on Oculyzer software version 1.20r20.

Patients were referred to Optegra for enhanced diagnosis with or without clinical suspicion of KC.

The data were collected in an Excel Sheet (Microsoft Corporation) and for statistical analysis Statistica 12.0 (StatSoft Polska) was used. The Shapiro-Wilk test was used for the evaluation of the distribution of continuous variables. Non-normally distributed variables are presented as median and range (minimum-maximum); normally distributed data are shown as mean \pm standard deviation. Categorical variables are shown as a percentage of the total number. Nonparametric Spearman correlation between analyzed parameters was calculated.

The study was approved by the ethics committee of Poznan University of Medical Sciences.

\section{RESULTS}

In total 73 patients (59 males and 14 females) were enrolled in the study, and data of 146 eyes were analyzed. Data of patients' age and coexisting atopy are presented in Table I.

Median UCVA was 0.2 and 0.6 LogMAR for the better and the worse eye respectively, and BCVA was 0.0 and 0.2 respectively. Further data of visual acuity are presented in Table II. Differences in visual acuity between the better and worse eye are statistically significant. Information about manifested refraction parameters are presented in Table II. Additional information about refractive error and astigmatism are presented in Table III.

The median grade was 1.5 for the better eye and 2.5 for the worse eye. Further corneal parameters - keratometry and TCC - are presented in Table IV. It has to be emphasized that differences in all discussed corneal parameters between the better and worse eye are statistically significant.

We counted the number of patients with BCVA 0.4 LogMAR or worse $(\geq 0.4)$ for the better and worse eye and found 3 patients (4.1\%) and $25(34.2 \%)$ respectively.

Numbers of eyes within defined $\mathrm{K}$ value ranges are presented in Table V.

Correlation between grade and cylinder (negative value) was calculated and a negative, statistically significant correlation was found: Spearman's $R=-0.327476, p=0.0002$. We also checked whether a correlation exists between grade

Table I. Number of patients by age, gender, and with atopy (\% of: all patients/ in subgroup)

\begin{tabular}{|l|c|c|c|c|} 
& $\begin{array}{c}\text { Median age } \\
\text { (range) }\end{array}$ & $\begin{array}{c}\leq 18 \text { years } \\
\text { old }\end{array}$ & $\begin{array}{c}>18 \text { years } \\
\text { old }\end{array}$ & Atopy \\
\hline Females & $25.3(16.1-43.5)$ & $1(1.4 / 20 \%)$ & $13(17.8 / 19.1 \%)$ & $3(4.1 / 13.0 \%)$ \\
\hline Males & $24.9(12.9-44.5)$ & $4(5.5 / 80 \%)$ & $55(75.3 / 80.9 \%)$ & $20(27.4 / 86.9 \%)$ \\
\hline
\end{tabular}


Table II. Visual acuity and refractive error: median value (range)

\begin{tabular}{|l|c|c|c|c|} 
& $\begin{array}{c}\text { All eyes } \\
(N=146)\end{array}$ & $\begin{array}{c}\text { Better eye } \\
(n=73)\end{array}$ & $\begin{array}{c}\text { Worse eye } \\
(n=73)\end{array}$ & $p$ value \\
\hline UCVA LogMAR & $0.39(-0.2-1.6)$ & $0.24(-0.2-1.5)$ & $0.62(0.2-1.6)$ & $<0.000001$ \\
\hline BCVA LogMAR & $0.12(-0.2-1)$ & $0.03(-0.2-0.4)$ & $0.24(0-1)$ & $<0.000001$ \\
\hline Sphere $n=110$ & $-1.0(-9-2.5)$ & $-0.9(-9-2.5)$ & $-1.0(-8-2.5)$ & 0.887 \\
\hline Cylinder $n=128$ & $-2.2(-6.5-0)$ & $-1.6(-4.3-0.5)$ & $-2.6(-6.5-0)$ & 0.00001 \\
\hline Axis $n=128$ & $90(0-175)$ & $95(12-170)$ & $88(0-175)$ & 0.350 \\
\hline
\end{tabular}

UCVA - uncorrected distance visual acuity, BCVA - best corrected distance visual acuity, $p$ - Mann-Whitney U test

Table III. Type of refractive error and type of astigmatism by the axis: number of eyes (\% of all eyes)

\begin{tabular}{|l|c|}
\hline Type of refractive error & Number of eyes \\
\hline Myopic compound astigmatism & \\
\hline Hyperopic compound or mixed astigmatism & $66(45.2 \%)$ \\
\hline Myopic astigmatism & $40(27.4 \%)$ \\
\hline Myopia & $22(15.1 \%)$ \\
\hline Hyperopia & $3(2.1 \%)$ \\
\hline Type of astigmatism by the axis & $1(0.7 \%)$ \\
\hline Against the rule $\left(a x i s: 61-119^{\circ}\right)$ & \\
\hline Oblique (axis: $\left.30-60^{\circ}, 120-150^{\circ}\right)$ & $74(50.7 \%)$ \\
\hline With the rule $\left(a x i s: 0-29^{\circ}, 151-180^{\circ}\right)$ & $33(22.6 \%)$ \\
\hline
\end{tabular}

value and age, with gender consideration. No statistically significant correlation was found (Spearman's $R=-0.061977$, $p=0.457$; Spearman's $R=-0.218153, p=0.265$; Spearman's $R=-0.016695, p=0.858$ for all patients, women and men respectively).

We also found that in 41 patients the left eye was the better eye, and in 32 patients the right eye.

\section{DISCUSSION}

As noted above, in recent studies reported $\mathrm{KC}$ prevalence is very high. Torres et al. found $\mathrm{KC}$ in nearly $5 \%$ of patients in the examined group, which consisted of pediatric patients from non-ophthalmic emergency departments [4]. In our study $5(6.9 \%)$ patients were $\leq 18$ years old. It has to be emphasized that $4(80.0 \%)$ patients in this subgroup were male. Awareness of $\mathrm{KC}$ prevalence in pediatric and adolescent patients should be increased, because visual impairment in this group may affect social and educational development. Moshiraf et al. recommend topographic screening in elementary schools as a way to provide early detection of $\mathrm{KC}$, due to the severity of the disease in children [32].
In the examined adult subgroup there were 55 (80.9\%) males and 13 (19.1\%) females. Our data from two age ranges demonstrate that a crucial difference in $\mathrm{KC}$ prevalence between genders exists. These results are similar to those published by Millodot et al. and Mohd-Ali et al. [3, 33]. The examined group of $1093 \mathrm{KC}$ patients in the study of Fink et al. included 482 (44\%) women and 611 men (56\%); mean age of the females was 40.0 years and mean age of males was 38.3 years $(p=0.01)$ [34]. In our study women are older than men, but the difference is not statistically significant. However, we suggest that usually KC patients are younger and male, and $\mathrm{KC}$ onset is later in female patients.

The most common refractive error in the examined group was compound myopic astigmatism; it was found in $66(45.2 \%)$ of examined eyes. We also found a high frequency of mixed astigmatism and hyperopic astigmatism (27.4\%), followed by myopic astigmatism (15.1\%). We found myopia in $2.1 \%$ of eyes and hyperopia in one eye. The most prevalent type of astigmatism was against-the-rule (50.7\%) and oblique astigmatism (22.6\%). Cruz-Becerril et al. reported higher prevalence of compound myopic astigmatism and with-therule astigmatism as dominant [21]. Using schematic eye models Tan et al. stated that cone location is the most important factor in vision distortion. $\mathrm{KC}$ cones cause myopia when they are located centrally. Peripherally located KC cones can result in hyperopic shift. The authors proved that one meridian of astigmatism will be aligned with the cone direction [35]. We did not evaluate the cone location. Our study is focused strictly on manifest refraction in KC, which is an important criterion in diagnosis and progression of KC. Refractive error examination in $\mathrm{KC}$ is a challenge and is often hampered by slight fluctuation in visual acuity at specific spherical power, cylinder power and axis.

Manifest astigmatism was greater in the worse than in the better eye and the difference between median values equated 1.0 D (absolute value), and was statistically significant. Based

Table IV. Corneal parameters: median value (range)

\begin{tabular}{|l|c|c|c|c|}
\hline Parameter & All eyes & Better eye & Worse eye & $p$ value \\
\hline Anterior K1 (D) & $44.2(39.6-56.2)$ & $43.1(39.6-52)$ & $45.4(40.2-56.2)$ & 0.000001 \\
\hline Anterior K2 (D) & $47.1(40.4-60.8)$ & $45.2(40.4-57.5)$ & $49.0(42.6-60.8)$ & $<0.000001$ \\
\hline TCC $(\mu \mathrm{m})$ & $473 \pm 40$ & $488 \pm 39$ & $458 \pm 34$ & $0.000001^{*}$ \\
\hline Grade & $2(0-4)$ & $1.5(0-3.5)$ & $2.5(0-4)$ & $<0.000001$ \\
\hline
\end{tabular}

Thinnest central cornea (TCC) - mean value $\pm S D, p-$ Mann-Whitney U test, *Student's t-test 
Table V. Number of eyes within defined K values ranges: number of eyes (\% of all eyes)

\begin{tabular}{|l|c|} 
& Number of eyes \\
\hline Anterior K2 $\leq 47.2 \mathrm{D}$ & $83(56.8 \%)$ \\
\hline Anterior K1 $\leq 43.0 \mathrm{D}$ & $53(36.3 \%)$ \\
\hline Anterior K2 $\leq 44.0 \mathrm{D}$ & $27(18.5 \%)$ \\
\hline
\end{tabular}

on this result we postulate that $1.0 \mathrm{D}$ (absolute value) intereye asymmetry in manifest astigmatism could be considered as a cut-off point for full $\mathrm{KC}$ screening with corneal tomography. Moreover, we found a statistically significant correlation between grade and amount of manifest astigmatism, which increases as the disease progresses.

Among various indices, keratometry has an important role in diagnosis, grading the disease and tracking its progression [31].

Yekta et al. analyzed K data from 2672 patients in a normal population and obtained the following results: mean flat meridian 42.98 D (95\% CI: 42.9-43.06 D) and mean steep meridian 43.98 D (95\% CI): 43.91-44.07 D) [36]. Even if K1 (flat) and $\mathrm{K} 2$ (steep) keratometry are within the normal range we always have to check inter-eye symmetry. In our group 36.3\% of $\mathrm{K} 1$ and $18.5 \%$ of $\mathrm{K} 2$ readings were within the normal range of values. Rabinowitz suggested several topometric criteria for the diagnosis of keratoconus, one of them being central $\mathrm{K}$ greater than $47.2 \mathrm{D}$ [37]. In our group $56.8 \%$ of eyes do not meet the requirements of this criterion.

Median values of $\mathrm{K} 1$ and $\mathrm{K} 2$ were higher in the worse eye, the difference between the eyes being statistically significant. Also the range of values was different, with higher values in the worse eye. Galletti et al. found that the mean anterior keratometry inter-eye difference $\geq 0.3 \mathrm{D}$ could be considered as a warning sign of $\mathrm{KC}$ [38].

We have to analyze pachymetry values similarly. The value of the better eye could be within the normal range, but comparison with the worse eye reveals inter-eye asymmetry [38]. The results of our study show significantly lower values of TCC in the worse eye $(458 \pm 34 \mu \mathrm{m})$ than in the better eye $(488 \pm 39 \mu \mathrm{m})$.

Galletti et al. stated that in nonkeratoconic eyes, the intereye asymmetry of thinnest pachymetry should be $\leq 12 \mu \mathrm{m}$. In our group it was $30 \mu \mathrm{m}$ [38].

Normal corneas are mostly symmetric, which is why the possibility of detecting ectatic disease by looking for inter-eye differences is especially valuable [39].

Another factor which is widely reported as coexisting with $\mathrm{KC}$ is atopy. Almost $32 \%$ of patients in our study reported atopy. It is similar to results published by Kaya et al., who found in the examined group of $70 \mathrm{KC}$ patients 33 patients with atopy [18]. Atopy may contribute to keratoconus but probably via eye rubbing associated with itching [40]. A significant relationship between the stronger dominant hand and the eye with more advanced keratoconus was proved in the McMonnies et al. study [41]. Although we did not check the dominant hand in our group, we found that the better eye was the left one in 41 cases - the right eye was the worse one in those cases, which correlates with data about hand dominance in the population - about $90 \%$ of people are right-handed [42].

Delay in proper diagnosis results in a decrease in the patient's visionrelated quality of life (VRQoL).

Best corrected visual acuity of the better eye is the foremost factor affecting VRQoL in patients with keratoconus [43]. In our study median BCVA of the better eye was 0.00 LogMAR but it has to be emphasized that $3(4.1 \%)$ patients functioned with BCVA 0.4 LogMAR and below. This value was reported as significantly lowering all aspects of the patient's VRQoL, e.g. distance vision, social functioning, and mental health [44]. Similar results, for treshold visual acuity 20/40, were published by Kymes et al. [45].

Patients with BCVA 0.4 and below in the worse eye reported significantly lower general health scores [44]. In our study there were $25(34.2 \%)$ patients with BCVA 0.4 and below in the worse eye.

There are limitations of this study. Our examined group is relatively small and we used only one set of diagnostic devices. It could be reasonable to validate our findings with other diagnostic systems.

\section{CONCLUSIONS}

We emphasize the importance of inter-eye asymmetry in $\mathrm{KC}$ screening and detection. We suggest suspecting $\mathrm{KC}$ during daily, routine practice in any case, even with normal BCVA and without pathological signs in basic ophthalmic examination, when: compound or mixed astigmatism is found with different manifest refraction values between eyes. Special vigilance should be exerted when inter-eye asymmetry in the amount of manifest astigmatism $\geq 1.0 \mathrm{D}$ (absolute value) exists. Any inter-eye asymmetry in keratometry or pachymetry values should be interpreted likewise. In these cases keratoconus diagnosis should be considered and full cornea diagnostics performed. Fast diagnosis prevents patients from functioning with lowered quality of life.

We can consider atopy as an additional criterion in screening of $\mathrm{KC}$.

We do not recommend analyzing any single value of any parameter derived from one eye as a cut-off criterion in $\mathrm{KC}$ diagnosis, e.g. there was a significant number of eyes with keratometry values within normal limits.

The incidence of keratoconus in patients below 18 years of age indicates that increased awareness of pediatric $\mathrm{KC}$ is needed. We postulate performing screening tests among adolescents to estimate the real prevalence of $\mathrm{KC}$, which is probably underestimated.

\section{DISCLOSURE}

The authors declare no conflict of interest. 


\section{References}

1. Rabinowitz YS. Keratoconus. Surv Ophthalmol 1998; 42: 297-319.

2. Perez-Straziota C, Gaster RN, Rabinowitz YS. Corneal cross-linking for pediatric keratcoconus review. Cornea 2018; 37: 802-809.

3. Millodot M, Shneor E, Albou S, et al. Prevalence and associated factors of keratoconus in Jerusalem: a cross-sectional study. Ophthalmic Epidemiol 2011; 18: 91-97.

4. Torres Netto EA, Al-Otaibi WM, Hafezi NL, et al. Prevalence of keratoconus in paediatric patients in Riyadh, Saudi Arabia. Br J Ophthalmol 2018: 102: 1436-1441.

5. Li X, Rabinowitz YS, Rasheed K, Yang H. Longitudinal study of the normal eyes in unilateral keratoconus patients. Ophthalmology 2004; 111: 440-446.

6. Wagner H, Barr JT, Zadnik K. Collaborative Longitudinal Evaluation of Keratoconus (CLEK) Study: methods and findings to date. Cont Lens Anterior Eye 2007; 30: 223-232.

7. Hutchings H, Ginisty H, Le Gallo M, et al. Identification of a new locus for isolated familial keratoconus at 2p24. J Med Genet 2005; 42: 88-94.

8. Tang YG, Rabinowitz YS, Taylor KD, et al. Genomewide linkage scan in a multigeneration Caucasian pedigree identifies a novel locus for keratoconus on chromosome 5q14.3-q21.1. Genet Med 2005; 7:397-405.

9. Gajecka M, Radhakrishna U, Winters D, et al. Localization of a gene for keratoconus to a 5.6-Mb interval on 13q32. Invest Ophthalmol Vis Sci 2009; 50: 1531-1539.

10. Shneor E, Millodot M, Blumberg S, et al. Characteristics of 244 patients with keratoconus seen in an optometric contact lens practice. Clin Exp 0ptom 2013; 96: 219-224.

11. Bral N, Termote K. Unilateral keratoconus after chronic eye rubbing by the nondominant hand. Case Rep Ophthalmol 2017; 8: 558-561.

12. Olivo-Payne A, Abdala-Figuerola A, Hernandez-Bogantes E, et al. Optimal management of pediatric keratoconus: challenges and solutions. Clin Ophthalmol 2019; 13: 1183-1191.

13. Najmi H, Mobarki Y, Mania K, et al. The correlation between keratoconus and eye rubbing: a review. Int J Ophthalmol 2019; 12: 1775-1781.

14. Lema I, Duran JA, Ruiz C, et al. Inflammatory response to contact lenses in patients iwth keratoconus compared with myopic subjects. Cornea 2008; 27:758-763.

15. Lema I, Sobrino T, Duran JA, et al. Subclinical keratoconus and inflammatory molecules from tears. Br J Ophthalmol 2009; 93: 820-824.

16. Mackiewicz ZMM, Stenman M, Konttinen L, et al. Collagenolytic proteinases in keratoconus. Cornea 2006; 25: 603-610.

17. Zadnik K, Barr JT, Edrington TB, et al. Baseline findings in the Collaborative Longitudinal Evaluation of Keratoconus (CLEK) Study. Invest Ophthalmol Vis Sci 1998; 39: 2537-2546.

18. Kaya V, Karakaya $M$, Utine $C A$, et al. Evaluation of the corneal topographic characteristics of keratoconus with orbscan II in patients with and without atopy. Cornea 2007; 26: 945-948.

19. Gordon-Shaag A, Millodot M, Shneor E, Liu Y. The genetic and environmental factors for keratoconus. Biomed Res Int 2015; 2015: 795738.

20. Farhood Q. Prevalence of refractive errors in patients with keratoconus among sample of Iraqi population. J Ophthalmol 2017; 2:2.

21. Cruz-Becerril A, Valdivia A, Peralta R, et al. Prevalence of refractive errors in Mexican patients with keratoconus. Clin Optom (Auckl) 2015; 7:39-44.

22. Serdarogullari $\mathrm{H}$, Tetikoglu $M$, Karahan $\mathrm{H}$, et al. Prevalence of keratoconus and subclinical keratoconus in subjects with astigmatism using pentacam derived parameters. J Ophthalmic Vis Res 2013; 8: 213-219.

23. Nemeth G, Szalai E, Berta A, Modis L Jr. Astigmatism prevalence and biometric analysis in normal population. Eur J Ophthalmol 2013; 23: 779-783.

24. Shakir AN, Alwan EH. Prevalence of keratoconus in patients with astigmatism more than two diopters: a cross-sectional study. Int J Med Invest 2019; 8: 12-22.

25. Reinstein DZ, Archer TJ, Gobbe M. Corneal epithelial thickness profile in the diagnosis of keratoconus. J Refract Surg 2009; 25: 604-610.

26. Silverman RH, Urs R, RoyChoudhury A, et al. Combined tomography and epithelial thickness mapping for diagnosis of keratoconus. Eur J Ophthalmol 2017; 27: 129-134.

27. Mohammadpour M, Heidari Z, Hashemi H. Updates on managements for keratoconus. J Curr Ophthalmol 2017; 30: 110-124.

28. Wollensak G. Crosslinking treatment of progressive keratoconus: new hope. Curr Opin Ophthalmol 2006; 17: 356-360.

29. Meek KM, Hayes S. Corneal cross-linking - a review. Ophthalmic Physiol Opt 2013; 33: 78-93.

30. Raiskup-Wolf $F$, Hoyer A, Spoerl E, Pillunat LE. Collagen crosslinking with riboflavin and ultraviolet-A light in keratoconus: long-term results. J Cataract Refract Surg 2008; 34: 796-801.

31. Gomes JA, Tan D, Rapuano CJ, et al. Global consensus on keratoconus and ectatic diseases. Cornea 2015; 34: 359-369.

32. Moshirfar M, Heiland MB, Rosen DB, et al. Keratoconus screening in elementary school children. Ophthalmol Ther 2019; 8: 367-371.

33. Mohd-Ali B, Abdu M, Yaw CY, Mohidin N. Clinical characteristics of keratoconus patients in Malaysia: a review from a cornea specialist centre. J Optom 2012; 5: 38-42.

34. Fink BA, Wagner H, Steger-May K, et al. Differences in keratoconus as a function of gender. Am J Ophthalmol 2005; 140: 459-468

35. Tan B, Baker K, Chen YL, et al. How keratoconus influences optical performance of the eye. JVis 2008; 8: 13.

36. Yekta, AA, Khabazkhoob M, Hashemi H, et al. The distribution of keratometry in a population based study. Acta Ophthalmol 2019; 97.

37. Rabinowitz YS. Videokeratographic indices to aid in screening for keratoconus. J Refract Surg 1995; 11: 371-406.

38. Galletti JD, Ruiseñor Vázquez PR, Minguez N, et al. Corneal asymmetry analysis by pentacam scheimpflug tomography for keratoconus diagnosis. J Refract Surg 2015; 31: 116-123.

39. Falavarjani KG, Modarres M, Joshaghani M, et al. Interocular differences of the Pentacam measure- ments in normal subjects. Clin Exp Optom 2010; 93: 26-30

40. Bawazeer AM, Hodge WG, Lorimer B. Atopy and keratoconus: a multivariate analysis. Br J Ophthalmol 2000; 84: 834-836

41. McMonnies CW, Boneham GC. Keratoconus, allergy, itch, eye-rubbing and hand-dominance. Clin Exp 0ptom 2003; 86: 376-384.

42. de Kovel CGF, Carrión-Castillo A, Francks C. A large-scale population study of early life factors influencing left-handedness. Sci Rep 2019; 9:584.

43. Sahebjada S, Fenwick EK, Xie J, et al. Impact of keratoconus in the better eye and the worse eye on vision-related quality of life. Invest Ophthalmol Vis Sci 2014; 55: 412-416.

44. Kurna SA, Altun A, Gencaga T, et al. Vision related quality of life in patients with keratoconus. J Ophthalmol 2014; 2014: 694542

45. Kymes SM, Walline JJ, Zadnik K, Gordon MO; Collaborative Longitudinal Evaluation of Keratoconus study group. Quality of life in keratoconus. Am J Ophthalmol 2004; 138: 527-535. 